\title{
Color Rapid Prototyping for Diffusion-Tensor MRI Visualization
}

\author{
Daniel Acevedo, Song Zhang, David H. Laidlaw, and Christopher W. Bull
}

\author{
Brown University, Providence, RI 02912, USA
}

We describe work toward creating color rapid prototyping (RP) plaster models as visualization tools to support scientific research in diffusion-tensor (DT) MRI analysis. We currently give surgeons and neurologists virtual-reality (VR) applications to visualize different aspects of their brain data, but having physical representations of those virtual models allows them to review the data with a very robust, natural, and fast haptic interface: their own hands. Our initial results are encouraging, and end users are excited about the possibilities of this technique. For example, using these models in conjunction with digital models on the computer screen or VR environment provides a static frame of reference that helps keep users oriented during their analysis tasks.

$\mathrm{RP}$ has been used in visualization largely for building molecular models to test assembly possibilities [1]. Nadeau et al. [4] created models of the human brain surface with the same RP techniques we use. Our approach, however, enables us to build inner brain structures.

\section{Method}

We examine the geometric models generated from a DTI dataset using tractography $[5,2,6]$. In our models, red streamtubes represent the diffusion in regions of linear anisotropy, where water diffuses primarily in one direction [6]. The streamtube direction represents the principal direction of diffusion along the tube. Studies show a correlation between the structures of neural fibers in white matter and the tracts derived from the principal direction of diffusion in linear

a)

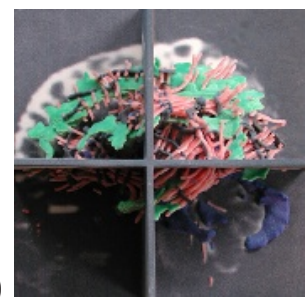

b)

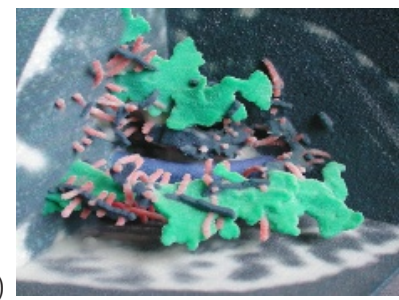

c)

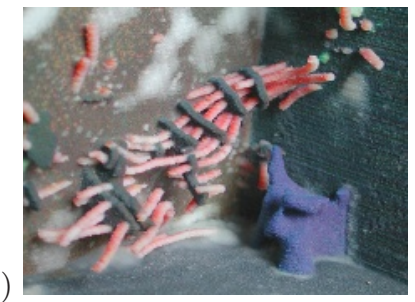

Fig. 1. (a,b) A plaster model showing areas of linear and planar water self-diffusion obtained from DT-MR images. (c) Detail of support structures (dark gray surfaces around tubes) for the streamtubes; these surfaces are created using the second and third eigenvectors of the tensors that produce the tubes. 
anisotropy regions [3]. Green streamsurfaces are generated in regions of planar anisotropy, where water diffuses primarily in a small plane at any given point. These planar structures could result from crossing fibers or laminar structures [6]. In addition to tubes and surfaces, we show anatomical features for context: blue surfaces show ventricles, and the images on the three orthogonal planes show slices of T2-weighted images collected with DTI.

To create our color models we use Z-Corp's Z406 printer. The digital model, in VRML format, is subdivided into horizontal layers by the printer software. These layers are then manufactured by putting down a thin layer of plaster powder and dropping colored binder at the boundaries of the model at that level. Once all the layers are built, the powder outside the boundaries of the model is vacuumed out and loose powder is removed using a fine blower. Finally, the piece is bathed in hot wax to strengthen it and enhance its colors.

The structures in the DT-MRI models require very careful treatment. Because the long thin streamtubes often fail to support themselves during powder removal, we inserted some supporting surfaces that interconnect neighboring streamtubes without occluding interesting features. These supports are created from the second and third eigenvectors of the diffusion tensor that creates the streamtubes, so they are perpendicular by definition (see Figure 1(c)). We arrived at this methodology after several tests, including building thicker tubes and increasing their number so they supported one another. Using information already present in the DT-MRI data, we have been able to create models with better structural stability.

\section{Results and Conclusions}

These early stages of development have highlighted some important issues. For example, our visualizations involve organic, free-form shapes, whereas current RP technology is designed for models with more regular shapes, such as mechanical parts and molecular models. Also, the printing and cleaning process can take as much as 12 hours for complicated brain models measuring up to $8^{\prime \prime} \times 8^{\prime \prime} \times 10^{\prime \prime}$. However, our initial experiments suggest that this technology has the great advantage of exploiting users' familiarity with physical models: they recognize the utility of holding them in their hands when studying them. Providing scientists with these models enhances the use and analysis of their digital counterparts. To quote one of the doctors who experimented with these models: "These physical models complement displays in digital format by providing a hard-copy frame of reference that can be touched and manipulated with optimum hand-eye coordination and immediate results." We believe this type of physical model can be very useful in preoperative planning when used as a quick reference for structure identification.

Acknowledgments. DT-MRI data provided by Dr. Susumu Mori (Johns Hopkins U.) Support from NSF (CCR-0086065, CCR-0093238) and Human Brain Project (NIDA, NIMH). 


\section{References}

1. M.J. Bailey et al. Curr. Op. Str. Bio., 8(2), 1998.

2. P.J. Basser et al. MR in Medicine, 44:625-632, 2000.

3. E.W. Hsu et al. Am. J. Physiol, 274, 1998.

4. D.R. Nadeau et al. IEEE Visualization 2000.

5. R. Xue et al. MR in Medicine, 42:1123-1127, 1999.

6. S. Zhang et al. IEEE TVCG, 9(4), 2003. 